\title{
B. Metal Ions in Drug Design, Neuroscience and Disease
}

\section{B-02}

\section{Interaction between Platinum Complexes and Copper Transport Proteins}

Giovanni Natile, Fabio Arnesano, Maurizio Losacco

Department of Farmaco-Chimico, University of Bari,

Bari 70125, Italy. natile@farmchim.uniba.it

Cisplatin ( cis- $\left.\left[\mathrm{PtCl}_{2}\left(\mathrm{NH}_{3}\right)_{2}\right]\right)$ and other platinum complexes used in anticancer therapy are able to cross the cell plasma membrane and eventually reach the nucleus where they form adducts with DNA which are responsible for the antitumor activity. Early studies suggested that cisplatin enters the cell mainly by passive diffusion; more recently, however, a growing body of evidence has revealed an alternative route for cisplatin uptake involving the copper transporter Ctr1. In a previous work we tested whether the extracellular domain of Ctr1 was also able to bind platinum complexes as a first step in the chemical mechanisms of cellular transport of platinum anticancer drugs by this protein [1].

We have now extended the investigation to other copper-binding proteins present in the cytosol. One of these, the copper chaperone Atox1, has been found to translocate to the nucleus where it acts as a copper-dependent transcription factor. We wanted to test if the same protein could also bind cisplatin and function as a platinum carrier. We have found that, indeed, Atox 1 reacts with cisplatin, displacing the two chlorido ligands (ESI-MS) and anchoring the metal core with the two cysteines of a CXXC motif.

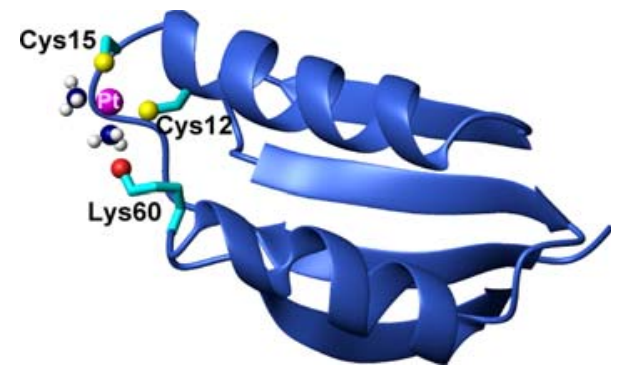

Reference

1. Arnesano F, Scintilla S, Natile G (2007) Angew Chem Int Ed 46:9062-9064

\section{B-03}

\section{Novel Metallopharmaceuticals for the Treatment} of Drug-Resistant Cancers and Infectious Diseases Ulrich Bierbach

Department of Chemistry, Wake Forest University, Winston-Salem, NC 27109, USA. bierbau@wfu.edu

This contribution summarizes the current status of a novel class of platinum-acridine hybrid agents developed to overcome several of the drawbacks associated with clinical platinum therapies. The prototypical compound, PT-ACRAMTU $[\mathrm{PtCl}(\mathrm{en})(\mathrm{ACRAMTU})]\left(\mathrm{NO}_{3}\right)_{2}$;
ACRAMTU $=1$-[2-(acridin-9-ylamino)ethyl]-1,3-dimethylthiourea), and its derivatives have demonstrated excellent activity in aggressive non-small cell lung cancer (NSCLC). The most promising analogue proves to be $\sim 25$-fold more potent than cisplatin in NCI-H460 lung cancer cells and slows the rate of $\mathrm{H} 460$ tumor growth in athymic nude mice by $40 \%$ relative to the control group. By comparison, cisplatin requires doses an order of magnitude higher to produce a similar effect in vivo. The new lead compound is the first non-crosslinking platinum agent endowed with promising activity in NSCLC in vivo.

As an extension of our platinum-intercalator technology, a new therapeutic approach is being pursued in which platinum-perylene conjugates are designed that target the G-quadruplex formed by the human telomeric repeat, [TTAGGG $]_{n}$. A chemical and biochemical overview of the nonclassical DNA interactions of these agents will be presented, and a critical evaluation will be made of their use as therapeutics and biochemical probes. Potential medicinal uses of novel gold(I) complexes derived from PT-ACRAMTU will also be discussed.

\section{B-04}

The Protective Role of $\mathbf{Z n}_{7}$ metallothionein-3

in Metal-linked Neurodegenerative Diseases

Milan Vašák ${ }^{1}$, Gabriele Meloni ${ }^{1}$ Andrea Crameri ${ }^{1}$, Günter Fritz ${ }^{2}$, Peter M.H. Kroneck ${ }^{2}$

${ }^{1}$ Department of Biochemistry, University of Zürich, 8057 Zürich, Switzerland.

${ }^{2}$ Fachbereich Biologie, Universität Konstanz, 78457 Konstanz, Germany.mvasak@bioc.uzh.ch

Aberrant interaction of $\mathrm{Cu}^{2+}$ with amyloid- $\beta(\mathrm{A} \beta)$, prion protein $(\operatorname{PrP})$ and $\alpha$-synuclein $(\alpha$-Syn) potentiate the progression of Alzheimer, prion and Parkinson diseases, respectively, by participating in the aggregation process and in the generation of reactive oxygen species (ROS). The production of ROS and neuronal toxicity are linked to the redox-cycling of copper. Very recently, we have showed that a small intra- and extracellularly occurring metalloprotein, metallothionein-3 ( $\left.\mathrm{Zn}_{7} \mathrm{MT}-3\right)$, by a metal swap between $\mathrm{Zn}_{7} \mathrm{MT}-3$ and the soluble and aggregated $\mathrm{A} \beta-\mathrm{Cu}(\mathrm{II})$ complexes abolishes the ROS production and the related cellular toxicity. In this process, $\mathrm{A} \beta-\mathrm{Zn}(\mathrm{II})$ and, following $\mathrm{Cu}^{2+}$ reduction by protein thiolates, a $\mathrm{Cu}(\mathrm{I})_{4} \mathrm{Zn}_{4} \mathrm{MT}-3$ species containing an air stable $\mathrm{Cu}(\mathrm{I})_{4}$-thiolate cluster and two disulfide bonds are formed. Our demonstration that $\mathrm{Zn}_{7} \mathrm{MT}-3$ in a similar reaction also removes $\mathrm{Cu}^{2+}$ from prion peptides and $\alpha$-Syn signifies a general protective role of this protein from $\mathrm{Cu}^{2+}$ toxicity in the brain.

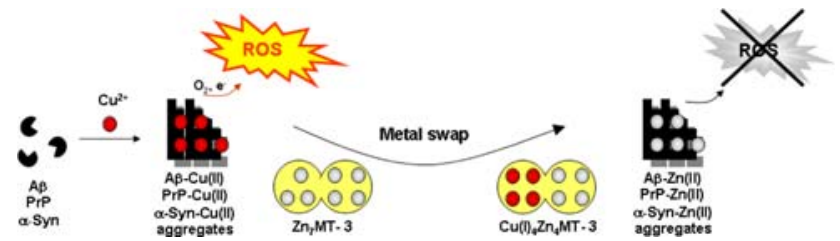




\section{B-05}

Nitrosyl [2Fe-2S] Proteins Models as New Nitric Oxide (NO) Donors for Chemotherapy

\section{Nataliya A. Sanina ${ }^{1}$}

${ }^{1}$ Institute of Problems of Chemical Physics, RAS, 1, Acad. Semenov Av., 142432, Chernogolovka, Russian Federation. sanina@icp.ac.ru Biological effects of NO have been associated with mechanisms that are life giving as well as toxic. With regard to cancer, various studies have demonstrated roles for NO in the induction of genotoxic lesions as well as its participation in tumor promotion and progression by mediating critical processes, including angiogenesis, tumor cell growth, and invasion. Yet $\mathrm{NO}$ is an important component of the immune response of some types of tumors. These properties stimulated activities to create antitumor drugs with a new mechanism of action, viz. NO-donors. Iron nitrosyl complexes are produced in reactions of endogeneous $\mathrm{NO}$ with active sites of non-heme [2Fe-2S] proteins and play important roles in organisms (cellular NO storage and transport). In this work a new stable crystalline nitrosyl complexes, namely $\left[\mathrm{Fe}_{2}(\mathrm{SR})_{2}(\mathrm{NO})_{4}\right]^{2-}, \quad\left[\mathrm{Fe}_{2}(\mathrm{SR})_{2}(\mathrm{NO})_{4}\right]$ and $\left[\mathrm{Fe}_{2}(\mathrm{SR})_{2}(\mathrm{NO})_{4}\right]^{2+}$ have been prepared [1]. It has been first found out that $\mathrm{NO}$ generated by nitrosyl $[2 \mathrm{Fe}-2 \mathrm{~S}]$ complexes can be considered as the efficient modulator of antitumor chemotherapy, and the compounds can be used as adjuvant means while using cytostatics. Cytotoxic efficacy of the complexes has been investigated on human tumor cell lines including K562 leukemia cells, MCF-7 breast carcinoma, A549 non-small-cell lung carcinoma, $\mathrm{SKOV}_{3}$ ovarian carcinoma. The antitumor activity of these complexes has been studied on the experimental tumors models (leukemia P388, adenocarcinoma Ca-755, melanoma B-16) [2]. These compounds are of interest for the practical medicine, i.e., for designing drugs of a new generation based on synthetic models of natural NO donors.

\section{References}

1. Sanina NA, Aldoshin SM (2004) Russ Chem Bull 11:2326-2345

2. Sanina NA, Zhukova OS, Smirnova ZS et al. (2008) Russ Biother J $1: 52$

\section{B-06}

\section{Interactions of Vanadium Compounds with Mitochondria}

Zhonglan Gao, Yuebin Zhao, Lihua Ye, Huixue Liu, Qin Xia, Yue zhang, Siwang Yu, Xiaoda Yang, Kui Wang

State Key Laboratories of Natural and Biomimetic Drugs and Department of Chemical Biology, School of Pharmaceutical Sciences, Peking University, 100191 Beijing, People's Republic of China. xyang@bjmu.edu.cn

Mitochondrion is a most important organelle involved in cellular signal crosstalking and ROS metabolism. Evidences suggested mitochondria an important target accumulating vanadium. To investigate the mechanisms of toxicity and/or potential pharmacological effects of vanadium compounds, we studied the effects of vanadium species on isolated mitochondria. The experimental results indicated that: (i) vanadyl ions bound to mitochondria with high affinity $\left(K_{d}=2.0 \times 10^{-16} \mathrm{M}\right)$. A vanadium-binding protein was isolated and identified to be the 60-kDa heat shock protein (Hsp60), an indispensable chaperone involved in the pathogenesis of inflammatory and autoimmune diseases e.g. type I diabetes; (ii) vanadium compounds $\left(\mathrm{VO}^{2+}, \mathrm{VO}_{3}{ }^{-}, \mathrm{VO}(\mathrm{acac})_{2}\right.$ and VOcit) (1-100 mM) induced mitochondrial swelling in a concentration-dependent manner and disruption of mitochondrial membrane potential $\left(\Delta \psi_{\mathrm{m}}\right)$ with a lap time, accompanied with elevated levels of reactive oxygen species (ROS). Cyclosporin A and antioxidants could significantly inhibit vanadium-induced $\Delta \psi_{\mathrm{m}}$ disruption. These results suggest that vanadium-induced oxidative stress caused a gradual opening of mitochondrial permeation transition pore (PTP), resulting in release of cytochrome $\mathrm{c}$ corresponding to the decrease of viability and cell apoptosis; (iii) certain vanadyl complex, i.e. VOdipic, could inhibit $\mathrm{Ca}^{2+}$-induced PTP opening, suggesting VOdipic as a novel PTP inhibitor. In summary, vanadium compounds could exhibit multiple actions on mitochondria and vanadium-induced oxidative stress on mitochondria could be associated to vanadium cytotoxicity.

Acknowledgement Supported by NSFC (\#20671008 \& \#20637010).

\section{B-07}

\section{SOD1 Fibrillation Mechanism Relevant to Familial Form of ALS: Molecular Dissection of Mutation- dependent Pathogenicity \\ Yoshiaki Furukawa, Kumi Kaneko, Nobuyuki Nukina}

Laboratory for Structural Neuropathology, Brain Science Institute, RIKEN, Wako, Saitama 351-0198, Japan. furukawa@brain.riken.jp Dominant mutations in $\mathrm{Cu}, \mathrm{Zn}$-superoxide dismutase (SOD1) cause a familial form of amyotrophic lateral sclerosis (fALS); selective degeneration of upper and lower motor neurons leads to paralysis and death. While accumulation of insoluble SOD1 in the affected tissues is a common pathological hallmark of SOD1-related fALS, it remains unknown why some disease phenotypes such as severity are different among the fALS-causing mutations. A wild-type SOD1 polypeptide becomes enzymatically active after post-translational modifications including copper/zinc binding and intramolecular disulfide formation [1]. These post-translational modifications also play an inhibitory role in the formation of insoluble SOD1 aggregates [2], which is evidenced by our findings that only the apo and disulfide-reduced state is accessible to fibrillar aggregation [3]. We also previously reported that fALS mutations increase intracellular fractions of the apo and disulfide-reduced SOD1 and thereby facilitate the formation of aggregates in cells [3]. Recently, we have noticed that aggregates of SOD1 with fALS-mutations are distinct in its morphologies as well as biochemical properties. Among fALS-mutant SOD1s, furthermore, a region(s) responsible for aggregation is found to be different. Our studies thus imply a new pathomechanism of fALS in which mutation-dependent properties of SOD1 aggregates modulate degree of disease phenotypes.

\section{References}

1. Furukawa $Y$ et al. (2004) EMBO J23:2872-2881

2. Furukawa $Y$ et al. (2006) Proc Natl Acad Sci USA 103:7148-7153

3. Furukawa Y et al. (2008) J Biol Chem 283:24167-24176 


\section{B-08}

\section{Chelation-based Approaches to Anticancer Drug Development}

\section{Jonathan L. Sessler}

Department of Chemistry and Biochemistry, 1 University StationA5300, The University of Texas, Austin, TX 78712-0165, USA. sessler@mail.utexas.edu

Mechanistic studies of motexafin gadolinium (MGd, 1) revealed that this well-studied experimental agent increases intracellular free zinc levels in several cell lines. Free zinc has been proposed to stabilize hypoxia-inducible factor-1 (HIF-1) and thus influence processes such as glycolysis, apoptosis, and angiogenesis. Moreover, free zinc inhibits thioredoxin reductase, a key mediator in the cellular response to oxidative stress that is frequently over-expressed in cancer. The ability of MGd to disrupt cellular zinc homeostasis led us to the consideration that small molecules capable of modulating free zinc levels would represent a new approach to anticancer lead generation. Initial studies have focused on the synthesis and study of solubilized pyrithione agents (e.g., 2 and 3) [1]. These systems show promise as anticancer leads, as judged from preliminary in vitro and/or in vivo analyses. We thus suggest that the present perturbation-based approach could be extended to other cations subject to homeostasis, including iron.
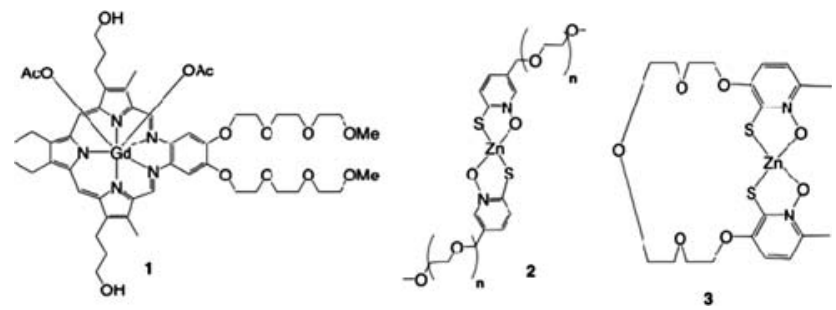

This work was supported by the NIH (CA 68687). A long-term collaboration with Dr. Darren J. Magda is also gratefully acknowledged.

\section{Reference}

1. Magda D, Lecane P, Wang Z, Hu W, Thiemann P, Ma X, Dranchak PK, Wang X, Lynch V, Wei W, Csokai V, Hacia JG, Sessler JL (2008) Cancer Res 68:5318-5325

\section{B-09}

\section{Design and Synthesis of Apoptosis-Inducing Agents Utilizing Intracellular Metal Cations}

\section{Shin Aoki}

Faculty of Pharmaceutical Sciences, Tokyo University of Science, Noda 278-8510, Japan. shinaoki@rs.noda.tus.ac.jp

$\mathrm{Zn}^{2+}$ is recognized as one of the most important cations in catalytic centers and structural cofactors of $\mathrm{Zn}^{2+}$-containing enzymes and DNA-binding proteins. Although most $\mathrm{Zn}^{2+}$ is tightly bound to enzymes and proteins, free zinc pools exist in some tissues such as brain, pancreas, liver, and prostate. It has also been revealed that $\mathrm{Zn}^{2+}$ is one of important cofactors in the regulation of apoptosis by a study utilizing $\mathrm{Zn}^{2+}$ fluorescent sensors [1-4].

In this presentation, the detection of early-stage apoptotic cells utilizing fluorescent $\mathrm{Zn}^{2+}$ probes will be presented [5, 6]. Our attempts at design and synthesis of apoptosis-inducing agents and inhibitors for protein-protein (and protein-DNA) interactions utilizing $\mathrm{Zn}^{2+}$ will also be described.

\section{References}

1. Zalewski PD, Truong-Tran AQ, Grosser D, Jayaram L, Murgia C, Ruffin RE (2005) Pharmacol Ther 105:127-149

2. Hashemi M, Ghavami S, Eshraghi M, Booy EP, Los M (2007) Eur. J. Pharmacol 557:9-19

3. Aoki S, Sakurama K, Matsuo N, Yamada Y, Takasawa R, Tanuma S, Shiro M, Takeda K, Kimura E (2006) Chem Eur J 12:9066-9080 4. Aoki S, Sakurama K, Ohshima R, Matsuo N, Yamada Y, Takasawa R, Tanuma S, Takeda K, Kimura E (2008) Inorg Chem 47:2747-2754

5. Kimura E, Aoki S, Kikuta E, Koike T (2003) Proc Natl Acad Sci USA 100:3731-3736

6. Kimura E, Takasawa R, Tanuma S, Aoki S (2004) Science STKE, p 17

\section{B-10}

Applications of X-ray Absorption Spectroscopy to the Studies of Metabolism of Biologically Active Transition Metal Complexes

Aviva Levina, Jade B. Aitken, Hugh H. Harris, Andrew I. McLeod, Zhi Jun Lim, Annanya Mitra, Annie Nguyen, Nguyen Pham, Anna Safitri, Lindsay E. Wu, Peter A. Lay School of Chemistry, University of Sydney, Sydney, NSW 2006, Australia. levina_a@chem.usyd.edu.au

Synchrotron-based techniques, including X-ray absorption spectroscopy and X-ray fluorescence mapping, are used as selective and sensitive tools for the studies of chemical transformations of transition metal complexes in single mammalian cells, bulk cell samples, and biological tissues and fluids. Here we present metabolic studies of carcinogenic $\mathrm{Cr}(\mathrm{VI})$ (see figure) [1], potentially anti-diabetic $\mathrm{Cr}(\mathrm{III})$, $\mathrm{V}(\mathrm{V} / \mathrm{IV})$ and $\mathrm{Mo}(\mathrm{VI})$ complexes [2, 3], and Ru(III) anti-cancer drugs. The first evidence of biological oxidation of $\mathrm{Cr}$ (III) nutritional supplements to potentially genotoxic $\mathrm{Cr}(\mathrm{VI} / \mathrm{V})$ species in adipocytes (fat cells) is also presented.

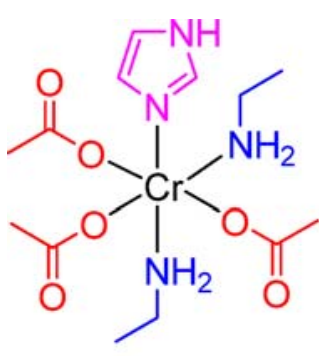

Cell Environment

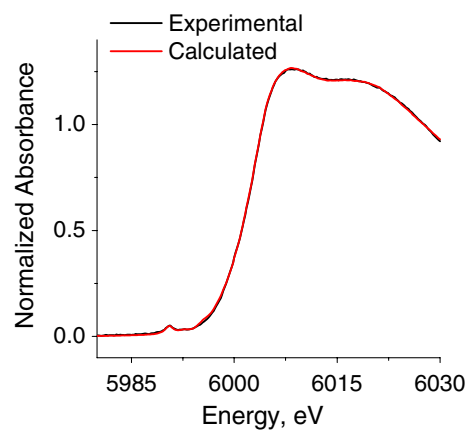

References

1. Levina A, Harris H, Lay PA (2007) J Am Chem Soc 129:10651075

2. Nguyen A, Mulyani I, Levina A, Lay PA (2008) Inorg Chem 47:4299-4309

3. Levina A, McLeod I, Seuring J, Lay PA (2007) J Inorg Biochem 101:1586-1593 


\section{B-11}

Gold(III)-based Anticancer Agents: Peptide Derivatives of Sulfur Donor Ligands as Improved Intracellular Drug Transfer and Delivery Systems Supported by Transporter Proteins

Luca Ronconi $^{1}$, Morelle Negom Kouodom ${ }^{1}$, Donatella Aldinucci ${ }^{2}$, Q. Ping $\mathrm{Dou}^{3}$, Fernando Formaggio ${ }^{1}$, Dolores Fregona ${ }^{1}$

${ }^{1}$ Department of Chemical Sciences, University of Padova, 35131 Padua, Italy.

${ }^{2}$ Department of Molecular Oncology and Translational Research, Division of Experimental Oncology 2, National Cancer Institute (CRO-IRCCS), Aviano (PN) I-33081, Italy.

${ }^{3}$ Ann Karmanos Cancer Institute and Department of Pathology,

School of Medicine, Wayne State University, Detroit, MI 48201,

USA. luca.ronconi@unipd.it

Only a few $\mathrm{Au}(\mathrm{III})$ compounds are currently under evaluation for their extremely promising antitumor properties. Recently, we have reported on some $\mathrm{Au}(\mathrm{III})$-dithiocarbamato derivatives which have proved to be much more cytotoxic in vitro than clinically established platinum-based drugs, and showed encouraging results in terms of both high in vivo effectiveness and lack of nephrotoxicity [1]. We have also identified the ubiquitin-proteasome system as a major in vitro and in vivo target [2], and evaluated their interaction with mitochondria [3], thus accounting for a different mechanism of action compared to cisplatin. We have now extended our research towards new $\mathrm{Au}$ (III)-dithiocarbamato derivatives of di-/tripeptides as improved intracellular drug transfer and delivery systems supported by transporter proteins PEPT1 and PEPT2, that mediate the cellular uptake of di-/tripeptides. As their substrate-binding site can accommodate a wide range of different molecules, they represent excellent targets for the delivery of pharmacologically-active compounds [4]. Synthesis, chemical characterization and biological activity are discussed.

\section{References}

1. Ronconi L et al. (2006) J Med Chem 49:1648

2. Ronconi L et al. (2006) Cancer Res66:10478

3. Ronconi L et al. (2007) Chem Biol 14:1128

4. Rubio-Aliaga I et al. (2002) Trends Pharmacol Sci 23:434

\section{B-12}

\section{A Computational Approach to Tuning the} Photochemistry of Pt(IV) Anticancer Agents

Hui-Chung Tai, Nicola J. Farrer, Anna E. Anastasi,

\section{Robert J. Deeth, Peter J. Sadler}

Department of Chemistry, University of Warwick,

Coventry CV4 7AL, UK. h-c.tai@warwick.ac.uk

$\mathrm{Pt}(\mathrm{IV})$ complexes are stable, inert pro-drugs which can be photoactivated to produce $\mathrm{Pt}(\mathrm{II})$ species which have promising anti-cancer activity. We show by TDDFT that the photochemistry of $\mathrm{Pt}(\mathrm{IV})$ complexes can be accurately modelled and therefore, that TDDFT can be used as a tool to help tune the coordination environment to achieve desirable photochemical properties. Exploration of trans influences, the nature of the axial and reducing ligands, and electron-withdrawing/donating substituents shows that the design of $\mathrm{Pt}(\mathrm{IV})$ complexes that exhibit greater absorption at longer wavelengths can be achieved. Absorption maxima can be red-shifted by up to $36 \mathrm{~nm}$ and the "tail" of the absorbance can be extended to $625-858 \mathrm{~nm}$. The ability to tune the photochemistry of this class of platinum compounds should be useful in optimizing their design as anticancer agents.

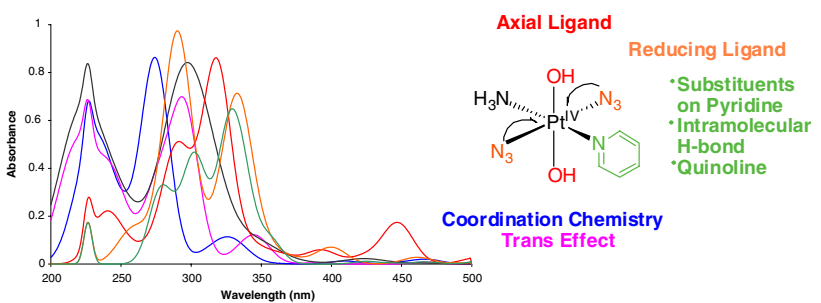

We thank EPSRC, MRC, WPRS and EC COST D39 for support.

\section{Reference}

1. Mackay FS, Woods JA, Heringova P, Kasparkova J, Pizarro AM, Moggach SA, Parsons S, Brabec V, Sadler PJ (2007) Proc Natl Acad Sci USA 104:20743-20748

\section{B-13}

How reliable are the "Working Hypotheses" that guide the field of $\mathrm{Pt}$ anticancer agents?

Yonit Kasherman ${ }^{1}$, Alina Nemirovski ${ }^{1}$, Inbal Vinograd ${ }^{1}$, Khuloud Takrouri $^{1}$, Yael Tzaraf ${ }^{2}$, Stefan Sturup ${ }^{2}$, Dan Gibson ${ }^{1}$

${ }^{1}$ Department of Medicinal Chemistry, Institute of Drug Research, School of Pharmacy, The Hebrew University, 91120 Jerusalem, Israel.

${ }^{2}$ Department of Pharmaceutics and Analytical Chemistry, Faculty of Pharmaceutical Sciences, University of Copenhagen, 2100 Copenhagen $\varnothing$, Denmark. dang@ekmd.huji.ac.il

Cisplatin, carboplatin and oxaliplatin are potent anticancer agents. They are believed to kill cancer cells by binding to the nuclear DNA and distorting its structure which triggers cellular processes that result in apoptosis. Most of the cellular Pt $(\sim 60 \%)$ is believed to be inactivated by glutathione (GSH) which binds irreversibly to the drug allowing only a fraction of the cellular Pt to bind to the DNA. Using ${ }^{15} \mathrm{~N}$ labeled cisplatin we looked at the interactions of cisplatin with cancer cell extracts and were not able to detect the formation of Pt-S bonds. Two dimensional NMR and analytical data (ICP-MS) suggest that most of the $\mathrm{Pt}$ is bound to cellular nucleophiles with MW $>3,000 \mathrm{Da}$ and not overwhelmingly to GSH.

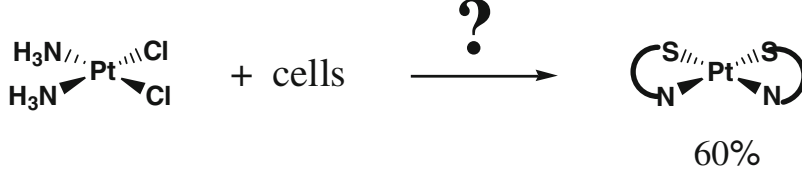

Some of the most active compounds are octahedral $\mathrm{Pt}(\mathrm{IV})$ prodrugs that are activated in the cells by reductive elimination whereby the two axial ligands are eliminated generating the cytotoxic $\mathrm{Pt}(\mathrm{II})$ complex. Two of the more interesting compounds, Satrplatin and LA12 , have acetato axial ligands. We have looked into the kinetics of the reduction of $\mathrm{Pt}(\mathrm{IV})$ complexes by cancer cells extracts and discovered that the reductive elimination of Pt(IV) complexes with axial acetato ligands can generate more than one product.

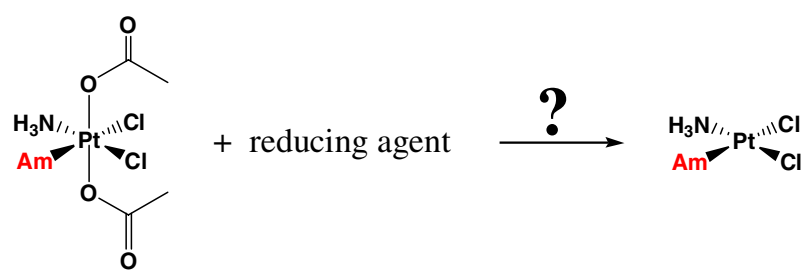




\section{B-14}

\section{Zinc is an intracellular signaling molecule.}

\section{Toshio Hirano}

Laboratory for Developmental Immunology and JST CREST Program, Graduate School of Frontier Bioscience, Graduate School of Medicine, and WPI Immunology, Frontier Research Centre, Osaka University and Laboratory for Cytokine Signaling, RIKEN RCAI, Osaka, Japan.hirano@molonc.med.osaka-u.ac.jp

Zinc ( $\mathrm{Zn})$ is an essential nutrient. $\mathrm{Zn}$ has been suggested to act as a kind of neurotransmitter. However, it had not been known whether $\mathrm{Zn}$ acts as an intracellular signaling molecule, i.e., a molecule whose intracellular status is altered in response to an extracellular stimulus, and that is capable of transducing the extracellular stimulus into an intracellular signaling event. We have proposed that there are at least two kinds of $\mathrm{Zn}$ signaling: "late $\mathrm{Zn}$ signaling," which is dependent on a change in the expression profile of $\mathrm{Zn}$ transporters, and "early $\mathrm{Zn}$ signaling," which involves a "Zn wave" and is directly induced by an extracellular stimulus [1]. (1) We showed that the nuclear localization of Snail is dependent on the $\mathrm{Zn}$ transporter, Slc39a6/Zip6/Liv1, a downstream target of STAT3 [2]. (2) Slc39a13/Zip13 is involved in TGF $\beta /$ BMP signaling because of its requirement for the nuclear localization of SMADs. It is required for connective tissue development and a responsible gene of EhlersDanlos syndrome [3]. (3) Toll-like receptor 4 (TLR4)-mediated dendritic cell maturation is at least in part dependent on a TLR4induced decrease in intracellular free zinc in a manner dependent on the change of expression profile of zinc transporters [4]. (4) FceR1stimulation induced an increase of intracellular free $\mathrm{Zn}$ and we named this phenomenon as "Zn wave" [5]. Zn wave seems to originate around ER and it occurs in a several minutes after the stimulation.

\section{References}

1. Hirano H et al. (2008) Adv. Immunol 97:149

2. Yamashita $S$ et al. (2004) Nature $429: 298$

3. Fukada $\mathrm{T}$ et al. (2008) PLoS ONE 3(11):e3642

4. Kitamura $\mathrm{H}$ et al. (2006) Nature Immunol 7:971

5. Yamasaki S et al. (2007) J Cell Biol 177:637

\section{B-15}

\section{Copper-Induced Oligomerization of Ubiquitin and $\alpha$-Synuclein}

Fabio Arnesano ${ }^{1}$, Vincenza Calò ${ }^{1}$, Maurizio Losacco', Simone Scintilla ${ }^{1}$, Giovanni Natile ${ }^{1}$

${ }^{1}$ Department of Farmaco-Chimico, University of Bari, 70125 Bari, Italy. arnesano@farmchim.uniba.it

Ubiquitin (Ub) plays a crucial role in intracellular protein degradation via the proteasome and the autophagy-lysosome pathways. Failure to eliminate misfolded proteins can lead to formation of toxic aggregates and cell death. Insoluble protein aggregates enriched with $\mathrm{Ub}$ are a hallmark of most neurodegenerative disorders including Parkinson's, Alzheimer's, amyotrophic lateral sclerosis, and prion diseases [1]. Metal ions have been implicated in the aggregation of disease-related, amyloidogenic proteins, however no report has focused on their possible role in the aggregation of Ub itself. We have found that $\mathrm{Cu}$ (II) binds to the $\mathrm{N}$-terminal end of $\mathrm{Ub}$ and promotes protein oligomerization into spherical particles [2]. By mimicking the condition of low dielectric medium experienced near a membrane surface, the assembly of spherical oligomers of Ub yields a series of intermediate species ending with a non-fibrillar filament network.
Annular intermediates and pore-like structures, stabilized by the interaction of $\mathrm{Cu}$ (II)-induced oligomers with lipid bilayers, resemble toxic protofibrillar species produced by several amyloidogenic proteins, such as Parkinson's $\alpha$-synuclein $(\alpha$-syn) [3, 4]. We also show that $\mathrm{Cu}$ (II) enhances oligomerization of $\alpha$-syn and formation of hybrid oligomers of $\alpha$-syn and Ub. Thus, we suggest that susceptibility to aggregation of $\mathrm{Ub}$ in the presence of $\mathrm{Cu}(\mathrm{II})$ ions may represent a potential risk factor for disease onset or progression while cells attempt to tag and process toxic substrates.

\section{References}

1. Ciechanover A, Brundin P (2003) Neuron 40:427

2. Milardi D, Arnesano F, Grasso G, Magrì A, Tabbì G, Scintilla S, Natile G, Rizzarelli E (2007) Angew Chem Int Ed 46:7993

3. Lashuel HA, Hartley D, Petre BM, Walz T, Lansbury PT (2002) Nature 418:291

4. Kayed R, Head E, Thompson JL, McIntire TM, Milton SC, Cotman CW, Glabe CG (2003) Science 300:486

\section{B-16}

Active Oxygen-Scavenging Activity of Manganese Salen Complexes in Water: Effect of a Three Dimensionally fixed Auxiliary

Yukinobu Noritake ${ }^{1}$, Yoritada Watanabe ${ }^{1}$, Azusa Namba ${ }^{1}$, Nobuki Kato $^{1}$, Naoki Umezawa ${ }^{1}$, Tsunehiko Higuchi ${ }^{1}$

${ }^{1}$ Graduate School of Pharmaceutical Sciences, Nagoya City University, 3-1 Tanabe-dori, Mizuho-ku, Nagoya 467-8603, Japan. higuchi@phar.nagoya-cu.ac.jp

Reactive oxygen species (ROS) often cause cell injury and have been implicated in a number of disease. Antioxidant therapies have been considered for a wide variety of disorders associated with oxidative stress. Synthetic catalytic scavengers of ROS would be clinically superior to stoichiometric ones. Among them, Salenmanganese complexes (Mn (Salen)) seem promising, because they exhibit dual functions, i.e. superoxide dismutase- and catalasemimic activities, and the reaction mechanism is thought to resemble that of catalase. Our approach was to increase the catalase-like activity of $\mathrm{Mn}(\mathrm{Salen})$ by arranging an appropriate auxiliary proximal to the $\mathrm{Mn}$ atom to create an environment resembling the interior of the active site of an enzyme. In order to fix the auxiliaries over $\mathrm{Mn}(\mathrm{Salen})$ plane at an appropriate position, we introduced a rigid bicycle[3,3,0]octane structure of which cyclopropane ring possesses an auxiliary at syn side to $\mathrm{Mn}(\mathrm{Salen})$. Catalase-like activities of prepared complexes were determined in aqueous phosphate buffer ( $\mathrm{pH}$ 7.4) by real-time monitoring of molecular oxygen concentration using a polarographic oxygen electrode at $25^{\circ} \mathrm{C}$. Ureido group-attached $\mathrm{Mn}$ (Salen) exhibited higher activity than conventional ones, probably due to its hydrogen peroxide recognition ability.

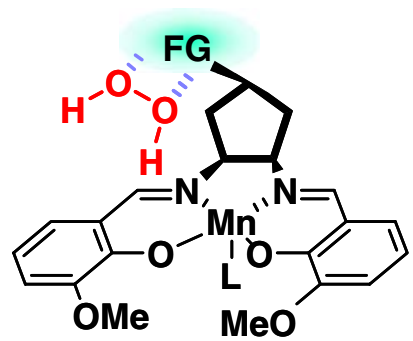




\section{B-17}

\section{Are Seven-Coordinate Manganese(II) SOD Mimetics} Selective towards Superoxide?

Ivana Ivanović-Burmazović ${ }^{1}$, Katharina Duerr ${ }^{1}$, Miloš Mojović ${ }^{2}$, Vlado Simović ${ }^{3}$, Robert Zimmerman ${ }^{3}$, Vesna Niketić ${ }^{4}$, Miloš R. Filipović ${ }^{\mathbf{1}}$

${ }^{1}$ Department of Chemistry and Pharmacy, University of ErlangenNürnberg, 91058 Erlangen, Germany. ${ }^{2}$ Department of Physical Chemistry, University of Belgrade, 11000 Belgrade, Serbia.

${ }^{3}$ Deparment of Transfusion Medicine and Hemostaseology, University Hospital Erlangen, University of Erlangen-Nürnberg, 91054 Erlangen, Germany. ${ }^{4}$ Department of Chemistry, University of Belgrade, 11000 Belgrade, Serbia.

Ivana.Ivanovic@chemie.uni-erlangen.de

Seven-coordinate $\mathrm{Mn}$ (II) pentaazamacrocyclic complexes represent the most potent synthetic mimics of native superoxide dismutase (SOD), which catalyze dismutation of superoxide $\left(\mathrm{O}_{2}^{\bullet-}\right)$ into $\mathrm{O}_{2}$ and $\mathrm{H}_{2} \mathrm{O}_{2}$ with efficiency that can exceed that of the mitochondrial MnSOD [1]. It is emphasized that the major advantage of $\mathrm{Mn}$ (II) pentaazamacrocycles over other SOD mimics is their high selectivity for $\mathrm{O}_{2}^{--}$and lack of reactivity with $\mathrm{NO}[1,2]$, a key molecule in biological processes. However, our studies have demonstrated that these complexes stimulate NO disproportionation via the catalytic mechanism based on the formation of labile metal-nitrosyl adducts and associated with $\mathrm{Mn}^{\mathrm{II}} / \mathrm{Mn}^{\mathrm{III}}$ redox cycle, in which metal bound NO exhibits the character and reactivity of $\mathrm{NO}^{-}$and $\mathrm{NO}^{+}$species. The concept of selectivity of $\mathrm{Mn}^{\mathrm{II}}$ pentaazamacrocyclic SOD mimics for $\mathrm{O}_{2}^{--}$and the lack of their reactivity towards $\mathrm{NO}^{2}$ is questioned by the present chemical and ex vivo study. We expect that biomedical implication of the present study will motivate further design and screening of truly selective SOD mimetics.

\section{References}

1. Salvemini D, Wang ZQ, Zweier JL, Samouilov A, Macarthur H, Misko TP, Currie MG, Cuzzocrea S, Sikorski JA, Riley DP (1999) Science 286:304-306

2. Salvemini D, Doyle TM, Cuzzocrea S (2006) Biochem Soc Trans 34:965-970

\section{B-18}

\section{Anti-Fenton Pro-chelators Triggered by Hydrogen Peroxide or Visible Light: A Novel Approach to Fight Neurodegenerative Diseases}

Yibin Wei, Maolin Guo

Department of Chemistry and Biochemistry, University of Massachusetts, Dartmouth, MA 02747, USA.mguo@umassd.edu Abnormal accumulation of redox metals (e.g., $\mathrm{Fe}$ and $\mathrm{Cu}$ ) in the brain and the metal-promoted Fenton reactions, which yield highly oxidizing and deleterious species, have been implicated in the pathogenesis of Alzheimer's and other neurodegenerative diseases [1]. $\mathrm{H}_{2} \mathrm{O}_{2}$, one of the key reactants in the Fenton reactions, is also a major endogenous Reactive Oxygen Species (ROS) [1]. Elevated levels of $\mathrm{H}_{2} \mathrm{O}_{2}$ and the Fenton reactions contribute to the oxidative stress and neurodegeneration. Anti-Fenton agents offer a promise in treatment of these diseases [1]. We have developed agents capable of doing "anti-Fenton reaction" via a $\mathrm{H}_{2} \mathrm{O}_{2}$-triggered prochelator activation and a subsequent metal caging strategy [2]. We have recently created our 2nd generation anti-Fenton fluorescent prochelators which are excitable [3] or triggerable by UV or visible light, thus allowing the anti-Fenton process to be controlled by light and monitored in real time by fluorescent techniques.
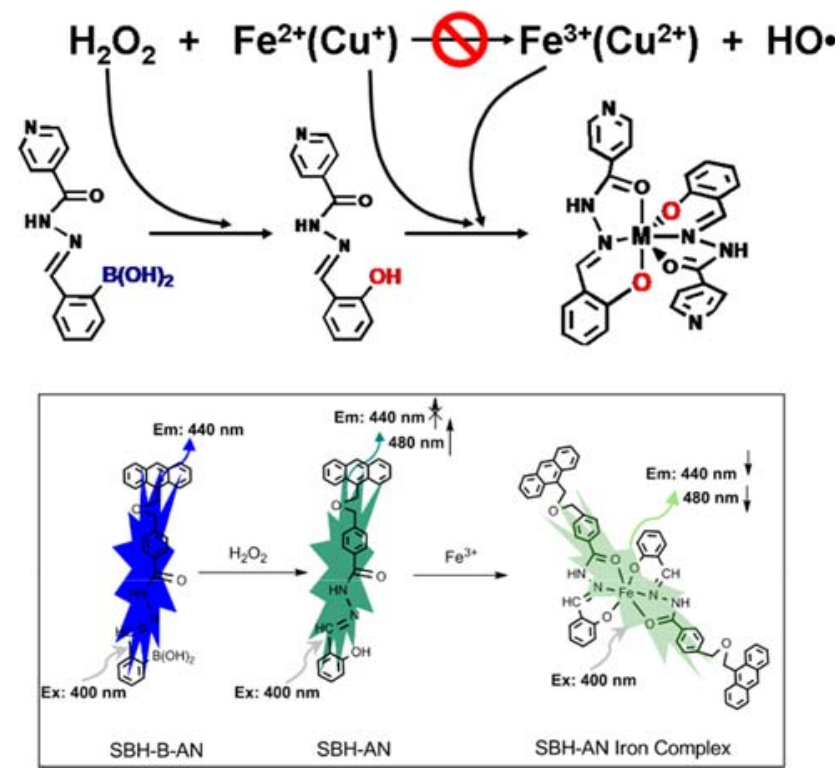

\section{References}

1. Perez CA, Tong Y, Guo M (2008) Curr Bioactive Comp 4:150-158

2. Wei Y, Guo M (2007) Angew Chem Int Ed 46:4722-4725

3. Wei Y, Guo M (2009) Chem Chem. doi:10.1039/b819204a, on-line

\section{B-19}

\section{Exploration of Non-platinum Metal Complexes}

\section{as Anticancer Agents}

Xiaoyong Wang*1, Zijian Guo ${ }^{2}$

${ }^{1}$ State Key Laboratory of Pharmaceutical Biotechnology, School of Life Sciences, Nanjing University, 210093 Nanjing, People's

Republic of China. ${ }^{2}$ State Key Laboratory of Coordination Chemistry, School of Chemistry and Chemical Engineering, Nanjing University, 210093 Nanjing, People's Republic of China. boxwxy@nju.edu.cn Platinum-based anticancer drugs play an important role in the treatment of various solid tumors. However, severe systemic toxicity and drug resistance have greatly hampered their continuing use and clinical efficacy. Therefore, it is necessary to develop new metal-based anticancer agents with different mechanisms of action. Non-platinum metal complexes provide a rich arsenal for seeking novel anticancer drugs; for example, complexes of ruthenium, gallium, and gold have shown impressive antitumor properties in preclinical studies. In recent years, we have synthesized various non-platinum metal complexes including those of palladium(II), gold(III), copper(II), bismuth(III), and nickel(II). Many of them demonstrate high cytotoxicity towards diverse tumor cell lines, especially those of 8-aminoquinoline derivatives (Fig. 1). Moreover, the antitumor mechanism of these complexes seems
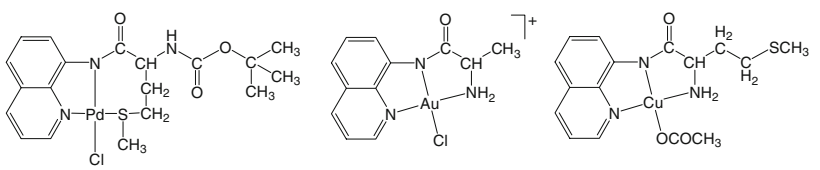

Fig. 1 Some antitumor active non-platinum metal complexes 
different from that of platinum complexes. The results indicate that the alteration of metal centers from platinum to non-platinum cation influences not only the spectrum of activity but also the toxic profiles and drug resistance properties of the complexes. For this reason, exploring the antitumor potential of non-platinum metal complexes is a promising option for the development of future anticancer agents.

\section{B-20}

Cu(II) Coordination of Alzheimer's Amyloid- $\beta$ Peptide: Site-selective Isotopic Labelling Studies using Multifrequency CW-EPR and HYSCORE spectroscopy Simon C. Drew ${ }^{1,2,3, *}$, Christopher J. Noble ${ }^{4}$, Colin L. Masters ${ }^{2,5}$, Graeme R. Hanson ${ }^{4}$, Kevin J. Barnham ${ }^{1,2}$

${ }^{1}$ Department of Pathology and Neuroproteomics Platform, National Neuroscience Facility, The Bio21 Molecular Science and Biotechnology Institute, The University of Melbourne, VIC 3010, Australia.

${ }^{2}$ Mental Health Research Institute, The University of Melbourne, VIC 3010, Australia.

${ }^{3}$ School of Physics, Monash University, VIC 3800, Australia.

${ }^{4}$ The University of Queensland, Centre for Magnetic Resonance and Centre for Metals in Biology, QLD 4072, Australia.

${ }^{5}$ Centre for Neuroscience, The University of Melbourne, VIC 3010, Australia.sdrew@unimelb.edu.au

Metal-induced $\mathrm{A} \beta$ oligomerisation is believed to play a key role in Alzheimer's disease [1]. Numerous X-band CW-EPR studies of $\mathrm{Cu}^{2+}$ interactions with monomeric $\mathrm{A} \beta$ have revealed two discernable species at physiological $\mathrm{pH}$, referred to as "component I" and "component II". The identity of the corresponding coordination modes has been controversial, with disagreement as to the role of Asp1, His6, His13 and His14. Using a library of A $\beta 16$ analogues with site-specific ${ }^{15} \mathrm{~N}$-labeling at Asp1, His6, His13, His14, we recently showed that component I is the sum of two coordination modes, $\left\{\mathrm{N}_{\mathrm{a}}^{\mathrm{D} 1}, \mathrm{O}, \mathrm{N}_{\varepsilon}^{\mathrm{H} 6}, \mathrm{~N}_{\varepsilon}^{\mathrm{H} 13}\right\}$ (component Ia) and $\left\{\mathrm{N}_{\mathrm{a}}^{\mathrm{D} 1}, \mathrm{O}, \mathrm{N}_{\varepsilon}^{\mathrm{H} 6}, \mathrm{~N}_{\varepsilon}^{\mathrm{H} 14}\right\}$ (component Ib), while component II comprises a $\left\{\mathrm{O}, \mathrm{N}_{\varepsilon}^{\mathrm{H} 6}, \mathrm{~N}_{\varepsilon}^{\mathrm{H} 13}\right.$, $\mathrm{N}_{\varepsilon}^{\mathrm{H} 14}$ \} coordination sphere [2]. The identity of the oxygen ligand(s) remains unclear, but ${ }^{17} \mathrm{O}$-labelling has ruled out Tyr10 [2]. Here we shed light on the nature of the oxygen coordination using site-selective ${ }^{13}$ C-labelling and HYSCORE spectroscopy.

\section{References}

1. Barnham KJ, Bush AI (2008) Curr Opin Chem Biol 12:222-228

2. Drew SC, Noble CJ, Masters CL, Hanson GR, Barnham KJ (2009)

J Am Chem Soc 131:1195-1207

\section{B-21}

\section{Improving Anticancer Drug Penetration in Solid Tumours}

Hambley, T.W., Bryce, N., Alderden, R.A., Whan, R.M., Yamamoto, N., Zhang, J.Z

t.hambley@chem.usyd.edu.au

The limited penetration of anticancer drugs into solid tumours is believed to be a major contributor to the failure of cancer chemotherapy.
We are developing strategies that make use of metal complexes to modify cellular and tumour uptake to give more effective treatment of all cells in a tumour and have been employing a combination of X-ray fluorescence mapping (SRIXE), XANES spectroscopy, and confocal fluorescence microscopy to monitor the uptake, metabolism, and activation of platinum(IV) and cobalt(III) prodrugs.

SRIXE mapping and XANES has allowed us to determine the location and oxidation state of platinum complexes in a variety of tumour models and has revealed significant problems with uptake into tumour spheroid models. These methods require access to synchrotron radiation and therefore we have been conducting parallel experiments using confocal fluorescence microscopy. Fluorescence tagging has proven to be an effective means for monitoring the cellular uptake, localisation, and site of action of these agents. Fluorescence microscopy has been employed to study the cellular distribution of several platinum-anthraquinone complexes by ourselves and others and has revealed that sub-cellular organelle localisation of such compounds is probably responsible for lower than expected activity. We have also shown that platinum and cobalt are able to alter the fluorescence of bound fluorophores and this has allowed us to monitor the coordination and oxidation state of both metals in cellular environments. Using changes in fluorescence, we have been able to visualise differences in uptake and distribution of the metal complexes and the fluorescent ligands. We have recently shown that we can modify the degree of penetration in models of solid tumours (spheroids) by variation of the metal complex charge.

\section{B-22}

\section{Multifunctional Ligands as Possible Interventions for Alzheimer's Disease}

Lauren E. Scott, Brent D. G. Page, Tim Storr, Brian O. Patrick, Chris Orvig

Medicinal Inorganic Chemistry Group, Department of Chemistry, University of British Columbia, 2036 Main Mall, Vancouver, BC V6T1Z1, Canada. orvig@chem.ubc.ca

Alzheimer's disease (AD) is the most common form of dementia. The amyloid hypothesis has been put forward to link observed pathologies in a causative manner. Specifically, dysfunctional interactions of aggregated amyloid-beta peptide and redox-active metal ions $(\mathrm{Cu}, \mathrm{Fe})$ are implicated in the development of highly oxidative conditions in the brain, leading to neurological decline. To potentially intervene in this process, small molecule metal ion-binding agents with antioxidant activity have been developed. Targeting to the brain through appended sugar moieties and via structural mimicry complete the multifunctionality of these new compounds. The synthesis of both the active and the glycosylated prodrug forms of the compounds, as well as the in vitro assay of their functionality will be discussed. 